\title{
Heat Load and its Effects on Fluid Friction Factor in Corrugated Pipes.
}

\author{
Ir. Peter Romeo Nyarko \\ Department of Mathematics and Statistics Kumasi Polytechnic \\ P.O. Box 854 Kumasi, Ghana, Email: romeoko2005@yahoo.co.uk
}

\begin{abstract}
In this paper, we investigate the effect of heat load on the fluid friction factor for laminar flow of a 2D axisymmetric straight corrugated pipe. Some clever assumptions are made on the NavierStokes equations to derive analytic expressions for computing the friction factor for the flow in terms of average velocity, density of the fluid, pressure drop and Reynolds number. The coupled momentum and energy equations are solved numerically and the effect of the heat load on the friction factor and hence the computed head loss in corrugated pipes/hoses analyzed. A new geometry is introduced for computing quasi-periodicity in pipes whose corrugations are periodically positioned. This has a positive effect of reducing instabilities in the solution and shows interesting features of the periodic pressure profile. Computational time and CPU memory have been drastically reduced as a result of simulating only one period as a true representation of an infinitely long pipe in which the flow is fully developed. We show that the heat load reduces the friction factor in corrugated pipes, this presupposes that pump requirements in the tropics are different from temperate regions for the same work done. The Moody Diagram shows a plot of the friction factor and Reynolds number at different corrugation heights without the effect of varying heat load. In this paper we show a diagram of the friction factor and Reynolds numbers at varying inlet and wall temperatures at a constant corrugation height. Furthermore we show that when flow is fully developed, periodizing the temperature on the boundary does not affect the flow.
\end{abstract}

Keywords: Axisymmetry, Corrugated pipe, Heat load, Quasi-periodicity, Laminar and turbulent flow, Momentum and Energy equations.

\section{INTRODUCTION}

In recent times, forced convection laminar flow inside a pipe has been at the fore front of research. The earliest formulation of the problem and analytical study can possibly be credited to Graetz (1885), who solved the problem of heat transfer to a constant property fluid in fully developed laminar flow in a pipe at constant wall temperature.

Since then several authors have studied the effect of heat load applied to many engineering problems. Wang and Vanka (1995) reported that there are higher values of friction factors for wavy channels to smooth wall channels of same internal spacing. Beer and Russ (1997) calculated mean friction coefficient per wavelength of axisymmetric pipe for three different amplitudes and reported frictional loses increased with increase of amplitude at the same Reynolds number. Van der Linden et. al. (2009) derived an efficient method to compute the friction factor in corrugated hoses.
The problem of heat transfer is of considerable interest in many engineering and industrial applications such as petroleum refinery, metal extrusion, glass fiber and paper production, water transport, polymer sheet extrusion from a dye, pipeline lubrication and processes in the chemical industry, Schlicting (2000).

In this paper we investigate the effect of heat load on the friction factor for an incompressible fluid flow in a corrugated pipe. The friction factor is an important ingredient in engineering design because it is used to calculate head lose and determining the pump pressure that drives the flow.

Normally for pipes to withstand high pressures, temperature and other forces, it has to be reinforced; therefore a fabric is wrapped over a spiral metal framework during the construction of such pipes. This reinforcement changes the internal geometry of the pipe causing corrugations that strongly affect the flow in the pipe. It promotes turbulence and improves heat transfer through such pipes. An important factor in 
pipe network design is to attain minimum pressure lose throughout the distribution lines and thus minimize the transportation cost.

To the best knowledge of the author there is no available chart on effect of heat load on the friction factor and Reynolds number as the one on the Moody Diagram. This means that at design stage, engineers would engage in numerous computations of head lose and pump pressure calculation in areas such as the tropics.

The investigation is organized as follows: In the first section, starting from the general Navier-Stokes equations governing fluid flow we derive exact solutions for the Hagan Poiseuille flow with constant fluid properties. Using the assumption of no swirl and axisymmetry of the pipe, the azimuthal component of the coordinate goes to zero. The idea of periodicity in pipe flow is employed to reduce computational cost and CPU memory to the order of $10^{6}$.

Our model is validated by comparing computations from the model to the experimental data on the Moody Diagram. Next is the introduction of a new simulation geometry for simulating quasi -periodicity. The new model geometry is set to have an upward flow to overcome the instabilities associated with pressure flows.

The governing equations and boundary conditions: The standard Navier-Stokes (N-S) equations are valid for laminar and turbulent flow modeling. In such flows the change from laminar to turbulent happen on such small length and time scales that direct numerical simulation becomes very expensive. The computational cost in terms of number of operations of direct numerical simulation is estimated at $\mathrm{Re}^{3}$ in Pitts et. al (1997). Therefore for a Reynolds number $(\mathrm{Re})$ of about one million, it becomes too costly on today's computer systems. For this reason we choose low Reynolds numbers so as to be in the laminar region. The three dimensional $\mathrm{N}-\mathrm{S}$ equations governing flows in cylindrical coordinates $(r, \theta, z)$ are given as:

Conservation of mass

$\frac{\partial \rho}{\partial t}+\frac{1}{r} \frac{\partial}{\partial r}\left(\rho r v_{r}\right)+\frac{1}{r} \frac{\partial}{\partial \theta}\left(\rho v_{\theta}\right)+\frac{\partial}{\partial z}\left(\rho v_{z}\right)=0$

Conservation of Momentum

$$
\begin{aligned}
& \frac{\partial v_{r}}{\partial t}+\left(v_{r} \frac{\partial v_{r}}{\partial r}+\frac{v_{\theta}}{r} \frac{\partial v_{r}}{\partial \theta}+v_{z} \frac{\partial v_{r}}{\partial z}-\frac{v_{\theta}^{2}}{r}\right)=-\frac{1}{\rho} \frac{\partial p}{\partial r}+v\left(\nabla^{2} v_{r}-\frac{v_{r}}{r^{2}}-\frac{2}{r^{2}} \frac{\partial v_{\theta}}{\partial \theta}\right) \\
& \frac{\partial v_{\theta}}{\partial t}+\left(v_{r} \frac{\partial v_{\theta}}{\partial r}+\frac{v_{\theta}}{r} \frac{\partial v_{\theta}}{\partial \theta}+v_{z} \frac{\partial v_{\theta}}{\partial z}+\frac{v_{r} v_{\theta}}{r}\right)=-\frac{1}{\rho} \frac{1}{r} \frac{\partial p}{\partial \theta}+v\left(\nabla^{2} v_{\theta}+\frac{2}{r^{2}} \frac{\partial v_{r}}{\partial \theta}-\frac{v_{\theta}}{r^{2}}\right) \\
& \frac{\partial v_{z}}{\partial t}+\left(v_{r} \frac{\partial v_{z}}{\partial r}+\frac{v_{\theta}}{r} \frac{\partial v_{z}}{\partial \theta}+v_{z} \frac{\partial v_{z}}{\partial z}\right)=-\frac{1}{\rho} \frac{\partial p}{\partial z}+v \nabla^{2} v_{z}-g
\end{aligned}
$$

\section{Conservation of Energy}

$\rho c_{p}\left(\frac{\partial T}{\partial t}+v_{r} \frac{\partial T}{\partial r}+\frac{v_{\theta}}{r} \frac{\partial T}{\partial \theta}+v_{z} \frac{\partial T}{\partial z}\right)=k\left[\frac{1}{r} \frac{\partial}{\partial r}\left(r \frac{\partial T}{\partial r}\right)+\frac{1}{r^{2}} \frac{\partial^{2} T}{\partial \theta^{\theta^{2}}}+\frac{\partial^{2} T}{\partial z^{2}}\right]+\mu \phi$

where $\phi$ is the dissipation function, $t$ is time, $p, v_{\theta}, v_{r}, v_{z}, v, \rho$ are pressure, azimuthal velocity, radial velocity, axial velocity, kinematic viscosity and the density of the fluid respectively, and $\mu$ is the dynamic viscosity. $T, k, c_{p}$ are temperature, coefficient of thermal conductivity and specific heat capacity of the fluid respectively. The governing equations are subject to the following boundary and inlet conditions. At the pipe wall or surface, the fluid is subject to the no slip condition:

$$
\mathrm{v}_{z}=0 ; \mathrm{v}_{r}=0 ; \mathrm{v}_{\theta}=0 .
$$

and one of the following thermal boundary conditions:

$$
\left\{\begin{array}{c}
q^{\prime \prime}=k \frac{\partial T}{\partial n}, \text { uniform heat flux } \\
T_{i}=T_{w}, \text { uniform wall temperature }
\end{array}\right.
$$

Finally, a uniform inlet velocity in the direction of flow

$$
v_{z}=v_{\text {in }}
$$

and uniform inlet temperature

$$
T_{\text {in }}=T_{0}
$$

Several laminar flow problems are encountered in the analysis of pipe flow.

In certain applications the velocity distribution may be developing if the pipe is not much longer than the hydrodynamic entrance length.

In such a case, the pressure drop will be much higher than the case where the velocity distribution is fully developed in a longer pipe. In other applications, especially when dealing with highly viscous fluids 
such as oils, the velocity profile develops more quickly than the temperature profile and may be considered to be fully developed in a region when the thermal boundary layer begins developing. Such problems are often referred to as Graetz problems or thermal entrance problems. The most general case (and difficult to analyze) occurs when both the velocity and temperature profiles develop together. This problem is generally referred to as the combined entrance problem or simultaneously developing flow. In this paper the fully developed velocity and temperature profiles are considered.

Analytical solution for the momentum and energy equations: The following assumptions are made on the governing equations to obtain analytic expressions for the velocity and temperature profiles, friction factor and the pressure drop in the pipe. The pipe under consideration has a diameter $(D)$, radius $(R)$, and $0 \leq r \leq R$. These assumptions are only valid to obtain analytic expression to the governing equations.

(i.) Steady incompressible flow, $\frac{\partial}{\partial t}=0$.

(ii.) Constant fluid properties

(iii.) No body force, $\bar{g}=0$.

(iv.) Negligible axial conduction

(v.) Negligible viscous dissipation

(vi.) Axisymmetric flow, $\frac{\partial}{\partial \theta}=0$.

(vii.) Flow is fully developed, $\frac{\partial}{\partial z}=0$.

(viii.) There is no swirl, $v_{\theta}=0$.

Under the above assumptions, the radial component of the velocity $\left(v_{r}\right)$ vanishes everywhere, this result implies that the streamlines of the flow are parallel to the wall of the pipe. To simplify the model, assume the pipe is horizontal, this makes the pressure gradient along the $z$-direction constant and therefore we can find the axial velocity $\left(v_{z}\right)$ from the axial momentum equation (0.4). $g_{r}=g_{z}=0$, and the sum of the first two terms on the right-hand side of equation (0.4) is equal to zero. This gives:

$$
-\frac{\partial p}{\partial z}+\mu \frac{1}{r} \frac{d}{\partial r}\left(r \frac{d v_{z}}{d r}\right)=0
$$

From equation (0.6) notice that the velocity component $v_{z}$ depends on the radius $(r)$ only therefore we introduce an arbitrary function $h(r)$ as the solution for $v_{z}$. In this way we can write equation (0.6) as:

$-\frac{\partial p}{\partial z}=\mu \frac{1}{r} \frac{d}{\partial r}\left(r \frac{d v_{z}}{d r}\right)=h(r)$

This implies

$p=h(r) z+C$

Similar analysis for the pressure variation in the radial direction, with our assumptions simplifies the momentum equation (0.2) as:

$\frac{\partial p}{\partial r}=0$

Thus all the terms in equation (0.2) goes to zero except the first term on the right-hand side. If we introduce a constant function $g(z)$ as integration constant, such that the pressure drop is a constant in the radial direction.

$$
p=g(z)
$$

Equation $(0.10)$ shows that the pressure gradient is constant. Equations (0.8) and (0.10) give two contradicting statements. On the one hand, the pressure $(p)$ is a function of the axial component only and on the other hand it is a function of the radius ( $r$ ) and axial component $(z)$.

This would be reconciled by requiring that $h(r)=C$. Therefore substituting this constant pressure gradient in equation (0.7) we obtain the parabolic-like axial velocity $\left(v_{z}\right)$ profile as:

$v_{z}=\frac{r^{2}}{4 \mu} \frac{d p}{d z}+C_{1} \ln (r)+C_{2}$

The logarithm of zero is negative infinity, therefore applying this boundary condition to $(0.11)$ at $r=0$ will imply the velocity will be infinite unless $C_{1}=0$, White (2003). 
This condition eliminates the possibility of a logarithmic singularity. Note that after the first integral of (0.7), we could apply this boundary condition, $r=0$ to the equation to obtain the same result that $C_{1}=0$. The no-slip boundary condition gives the constant $C_{2}$. Therefore the axial velocity is given by:

$v_{z}=-\frac{1}{4 \mu} \frac{d p}{d z}\left(R^{2}-r^{2}\right)$

Equation (0.12) is a parabolic velocity profile in the axial direction which shows that the maximum velocity of pipe flow is at the center $(r=0)$.

Fig 1 shows that at different pressure gradients the shape of the velocity profile in the radial direction is independent of the pressure gradient for a horizontal pipe.

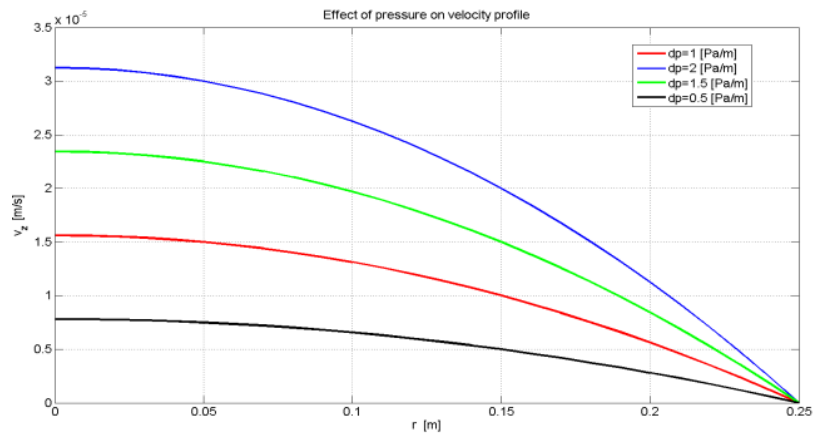

Fig 1: Velocity profile in the axial direction at $\frac{d p}{d z}=(0.5,1.0,1.5,2.0)$

Pressure drop and friction factor: The engineer is frequently interested in the pressure drop needed to sustain an internal flow because this parameter determines pump power requirement, Balasubramanian (2006). From the Hagan Poiseuille and the Darcy Weisbach equations we obtain two different expressions for the friction factor $(f)$ :

$$
\begin{aligned}
& f_{1}=\frac{64}{\operatorname{Re}} \\
& f=-\frac{4 R}{\rho \overline{v^{2}}} \frac{d p}{d z}
\end{aligned}
$$

Friction factors for a wide range of Reynolds number are presented in the Moody diagram. In addition to the Reynolds number, the friction factor is a function of the tube surface condition $f=F\left(\operatorname{Re}, \frac{\varepsilon}{D}\right)$, where $\varepsilon$ and $D$ are the roughness (corrugation) height and the diameter of the pipe respectively. The friction factor is minimum for smooth surface and increases with increasing surface roughness $(\varepsilon)$. Correlations that reasonably approximate the smooth surface condition are of the form:

$$
\begin{aligned}
& f=0.316 \operatorname{Re}_{D}{ }^{-1 / 4}, \operatorname{Re}_{D} \leq 2.0 \times 10^{4} \\
& f=0.184 \operatorname{Re}_{D}{ }^{-1 / 5}, \operatorname{Re}_{D} \geq 2.0 \times 10^{4}
\end{aligned}
$$

We do not investigate so much into the corrugations, details of the effect of corrugation on the friction factor can be found in Pisarenco (2007), van der Linden et. al. (2009), Mahmud et. al. (2003). We have shown that in the fully developed region the pressure gradient $\left(\frac{d p}{d z}\right)$ is a constant, therefore from equation $(0.14)$ the pressure drop for fully developed flow from two axial positions $\left(z_{1}\right.$ to $\left.z_{2}\right)$ can be found from:

$$
\Delta p=\int_{p_{0}}^{p_{1}} d p=f \frac{\rho \overline{v^{2}}}{2 D} \int_{z_{1}}^{z_{2}} d z=f \frac{\rho \overline{v^{2}}}{2 D}\left(z_{1}-z_{2}\right)
$$

If the Reynolds number and the pressure at two points are known, we can find the corresponding friction factor from the Moody diagram and subsequently compute the head loss and pump pressure for the flow.

Temperature profile: Forced convection heat transfer problems in pipe flow may be classified as type (I) thermally developing and type (II) thermally fully developed. The first type (thermally developing) may be further divided depending on whether the velocity profile is developing or fully developed. Simultaneous development of velocity and temperature profiles is very difficult to analyze. Most solutions to these type of problems have been obtained using numerical methods, Shah and London (1978). The temperature profile from equation (0.5) has been thoroughly discussed in Nyarko (2011) for the case where the hydrodynamic boundary layer is fully developed.

We only quote the parabolic temperature profile as: 
$T=T_{w}-2 P e \frac{d T_{m}}{d z}\left(\frac{3 R^{2}}{16}+\frac{r^{4}}{16 R^{2}}-\frac{r^{2}}{4}\right)$

where $T_{w}$ and $\mathrm{T}_{m}$ are the wall and mean temperatures respectively

$$
P e=\frac{v}{\alpha}
$$

The Peclet number $(P e)$ is the ratio of convected heat energy to conducted heat energy within the fluid. It is also the product of the Reynolds number and Prandtl number. A small Peclet number corresponds to dominant thermal conduction. Fig 2 shows the temperature profile of equation (0.18) along the axial direction for a pipe of radius $(R=0.2 \mathrm{~m})$ and length $(\mathrm{L}=1.0 \mathrm{~m})$, at different values of the dimensionless Peclet number $(P e=1,1.33,2)$. We note that if the liquid conducts less heat, the temperature profile is more parabolic. This supports the statement that if the velocity of the fluid in the pipe is high, it conducts less heat.

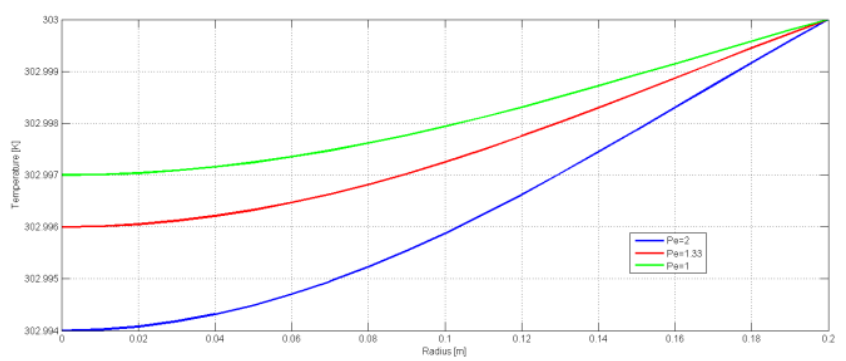

Fig 2: Temperature profile in the axial direction for ( $P e=1,1.33,2$ ), L=1.0m

Numerical simulations: The assumption of a fully developed flow allows us to state that for an infinitely long pipe, the flow will be periodically fully developed because of the periodic positioning of the corrugations Patanker et. al. (1977).

In such flows the velocity repeats itself at corresponding axial locations in successive circles. This allows us to consider only one period in our simulation as a true representation of what happens in a long corrugated pipe/hose. This idea coupled with the use of periodic boundary condition decrease computational cost and CPU memory.

Of course we do not have measurement data for the model that we simulate but to determine the degree to which the model is an accurate representation of the real world, we validate the model for specific range of application for which there is experimental data.

The Moody Diagram, Moody (1944) is a large data base of experimental data (confirmed by the theoretical Colebrooke-White equation). It represents graphically the friction factor as a function of Reynolds number. At low Reynolds numbers it shows the $\frac{64}{\mathrm{Re}}$ relationship for laminar flows. We first simulate the momentum equation in a smooth walled pipe using the finite element package Comsol Multiphysics, White (2008) without adding heat. The results of the computed friction factor from the simulations are compared to the one given by the Moody diagram.

Model and domain geometry: The laminar incompressible Navier-Stokes equations are used on a 2D axisymmetry pipe. The pipe diameter is $0.4 \mathrm{~m}$ and the pipe/hose is filled with a water-like liquid with speeds 5 to $10 \mathrm{~m} / \mathrm{s}$, density of $1000 \mathrm{~kg} / \mathrm{m}^{3}$ and a viscosity of $1 \mathrm{mPa}$.s. The Reynolds number is $R e=10^{3}$, (well in the laminar region).

The corrugation height is $0.01 \mathrm{~m}$, which is less than $3 \%$ of the diameter (ie. $\frac{\varepsilon}{D}=0.025$ ), therefore values on the Moody Diagram can be used to validate the model. The pipe length $(L)$ is $1.14 m$.

Smooth wall boundary conditions: The computational domain has four boundaries. On the right hand side boundary we prescribe the no slip boundary condition to the pipe wall. On the left hand side which coincides with the centerline of the pipe we prescribe axial symmetry boundary condition.

A parabolic velocity profile is prescribed at the inlet boundary and at the outflow boundary; we prescribe pressure equal to zero.

After solving the problem on a coarse and fine mesh using the adaptive solver, it was realized that the solution was mesh independent.

Fig 3 (a) shows the numerical results for the surface velocity field. The parabolic velocity profile along the radial direction at $z=0.1 \mathrm{~m}$ is maximum at $r=0$, confirming the analytical solution from equation ( 0.12$)$ and by the color legend in Fig 3 (a). We can observe 
that both Fig 3 (a) and Fig 3(b) show a maximum velocity value of $0.5 \mathrm{~m} / \mathrm{s}$.

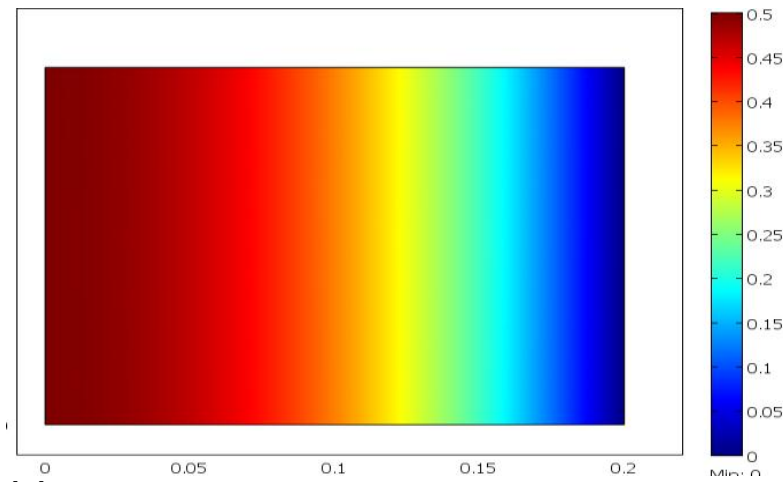

(a)

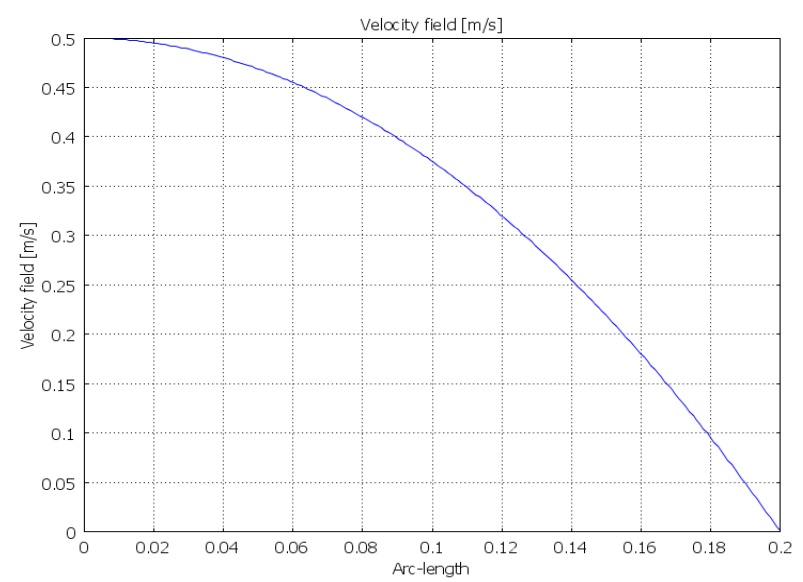

(b)

Fig 3 (a) surface velocity,(b) Velocity profile along the radial direction at $z=0.1 \mathrm{~m}$.

Fig 4(a) shows the computed friction factors from equations (0.13) in blue and (0.14) in red against Reynolds number. In Fig 4(b), the logarithmic plot of the computed friction factors from our model agrees with the Moody diagram with an error less than $1 \%$.

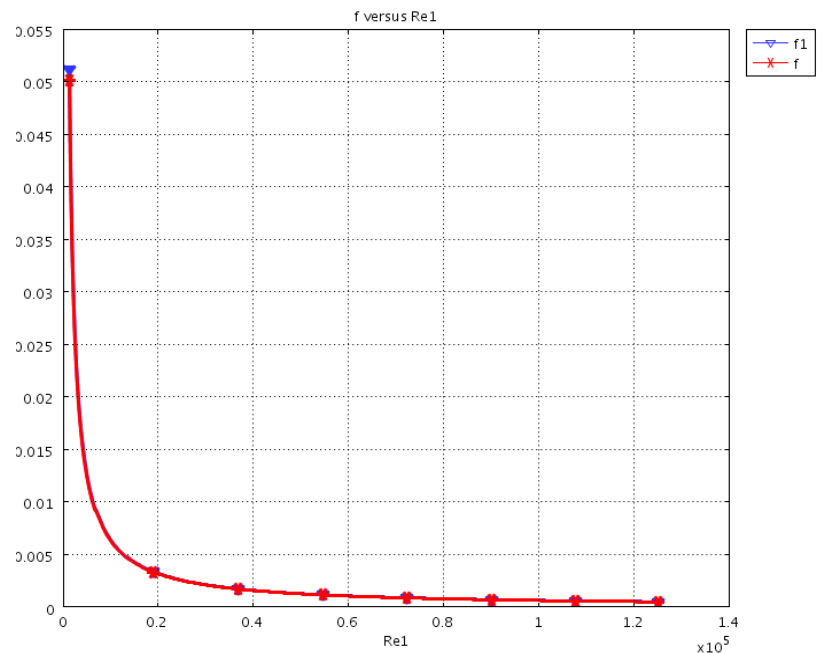

(a)

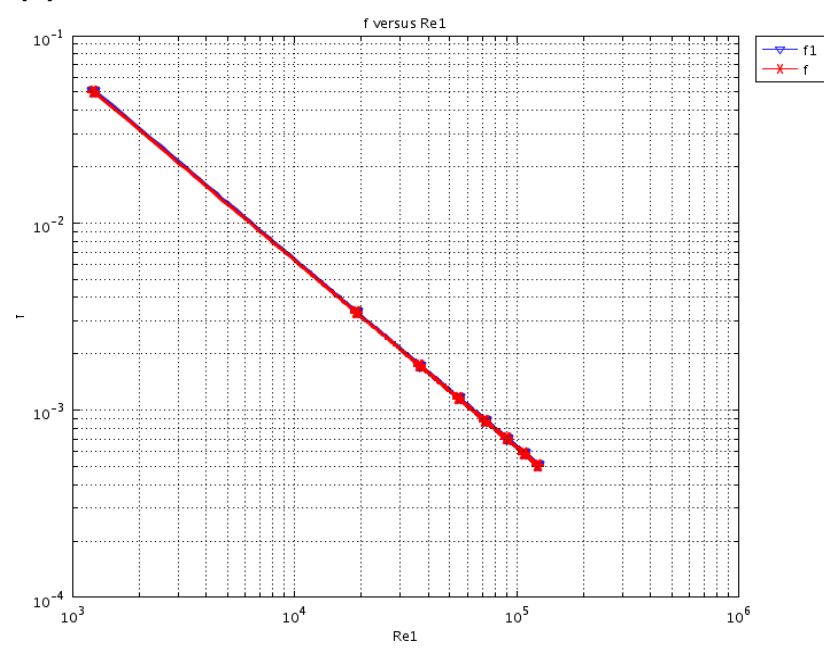

(b)

Fig 4: (a) Computed Friction factors Vrs Reynolds number, (b) Log plot of the computed friction factors Vrs Reynolds number.

Corrugated pipe model simulation: The flow in the corrugated pipe is different from that in the smooth pipe because the flow is immensely affected by the presence of adverse pressure gradients. As a result, the model is set to have an upward flow such that the negative pressure gradient overcomes the shear stress caused by viscosity and other forces which retards the flow. Details of the characteristics of pressure gradient on flow in a corrugated hose can be found in Pisarenco (2007), Beer and Russ (1997), White (2003). 
Simulation domain geometry: Fig 5 shows the new computational domain we have introduced for simulating quasi-periodicity for pipe flow. It includes three periods of the corrugations in the pipe. At the left and at the right, it is bounded by the symmetry axis and by the wall respectively, while the inflow/outflow boundaries are the ones located at the bottom and top of the domain with continuity on each boundary.

The coupled momentum and energy equations are solved simultaneously with appropriate boundary conditions. The new periodic modeling shown in Fig 5 called Pseudo-periodic is to take care of the instabilities usually associated with pressure flows with the assumption that the first and third periods take care of the instabilities whiles the solution in the second period is undisturbed. Therefore although there are three periods, we only consider the second (middle) domain. This model allows us to prescribe periodic boundary conditions. The problem is first solved by periodizing the velocity and pressure, considering the temperature on the wall and the inlet as variables.

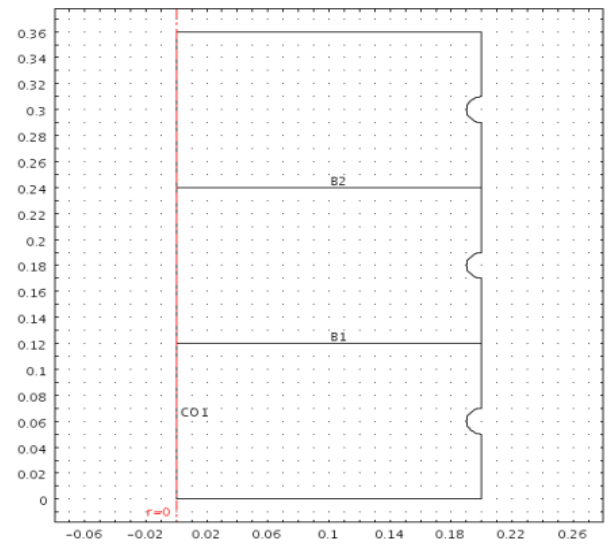

Fig 5: 2D axisymmetry pseudo-periodic model.

Boundary conditions: Unlike the smooth wall model, the 2D axisymmetric model shown above (Fig 5 ) has five boundaries on each domain and we prescribe boundary conditions to each. Taking $z=0$ to be anywhere in the pipe/hose where the flow can be assumed to be fully developed, we just need to simulate the flow computations between $0<z<L$, where $L$ is the length of the period. On the left boundary which coincides with the centerline of the pipe we prescribe axial symmetry boundary condition.

On the right hand side boundary, we have two materials, a steel spiral and fabric. Both surfaces are considered smooth and therefore the no slip boundary condition is prescribed on the wall.

The periodic boundary conditions for the velocity at the inlet and outlet boundaries are:

$U(r, 0)=U(r, L)$

$V(r, 0)=V(r, L)$

If the pressure at the boundaries labeled $B 1$ and $B 2$ of Fig 5 are $P_{0}$ and $P_{1}$ respectively, such that $P_{0}>P_{1}$ in Fig 5 , and $\Delta p=P_{0}-P_{1}$, then the pressure between these two points can be decomposed as:

$p(r, z)=-f_{z} z+p(r, z)$

where $f_{z} z$ is the static pressure and $p(r, z)$ is the periodic part of the pressure.

For periodicity at the inflow and outflow,

$$
p(r, 0)=p(r, L)
$$

The pressure drop is given by taking the difference in pressure between the two points to obtain:

$\Delta p=f_{z} L$

We can therefore write the periodic pressure boundary condition as,

$p\left(r, z_{\text {in }}\right)=p\left(r, z_{\text {out }}\right)+f_{z} L$

Boundary conditions are also prescribed for the energy equation therefore; on the left boundary which coincides with the centerline of the pipe we prescribe axial symmetry boundary condition. Whiles wall temperature is prescribed on the right side boundary consisting of two materials as stated above, (this temperature is varied in the simulation). At the inlet we prescribe the inlet/entry temperature of the fluid (This temperature is also varied in the simulation). Finally we prescribe convective flux on the outflow boundary.

Meshing: In fluid flow dynamics, constructing a mesh is of the same importance as using the correct equations. The adaptive solver in Comsol Multiphysics is used to construct the mesh because it has the quality of constructing the mesh and calculating the corresponding solution simultaneously. Adaptive mesh generation identifies 
Am. J. Sci. Ind. Res., 2012, 3(4): 241-251

the high-activity regions that require a high resolution (by estimating the errors) and produces an appropriate mesh.

Postprocessing: The following global variables and parameters that affect the flow and boundary conditions of the model are computed from the simulation.

Change in pressure at two points $z_{1}$ and $z_{2}$ :

$\Delta p=p_{0}-p_{1}$

Average velocity,

$\bar{U}=\frac{Q}{\pi R^{2}}$

Reynolds number,

$\operatorname{Re}=\frac{2 \rho \bar{U} R}{\mu}$

Friction factor,

$f=\frac{4 f_{z} R}{\overline{U^{2} \rho}}$

Numerical results: The plot in Fig 6 shows the computed friction factor verses Reynolds number for the thermally and hydrodynamically fully developed flow at equal inlet and wall temperature $\left(T_{i n}=T_{w}=60^{\circ} \mathrm{c}\right)$. Looking at Fig $4 \mathrm{a}$, and Figure 6 the friction factor seems to be decreasing with an increasing Reynolds number but the Reynolds numbers are different, that is $\operatorname{Re} \approx 1 \times 10^{4}$ and $\operatorname{Re} \approx 4.25 \times 10^{3}$ respectively for the same value of the friction factor ( $f=0.01$ ) on both figures.

The lower Reynolds numbers in Figure 6 is due to the effect of temperature on the flow such that the transition from laminar to turbulent flow occurs at lower Reynolds numbers (ie. $\operatorname{Re}<2000$ ). The change in gradient on Fig 6 is due to the change in viscosity as a result of the heat load on the flow. It is anticipated that the thin layer of fluid close to the pipe wall will have viscosity different from the bulk flow viscosity due to the heat load on the wall but this has not yet been proven and research is in progress.

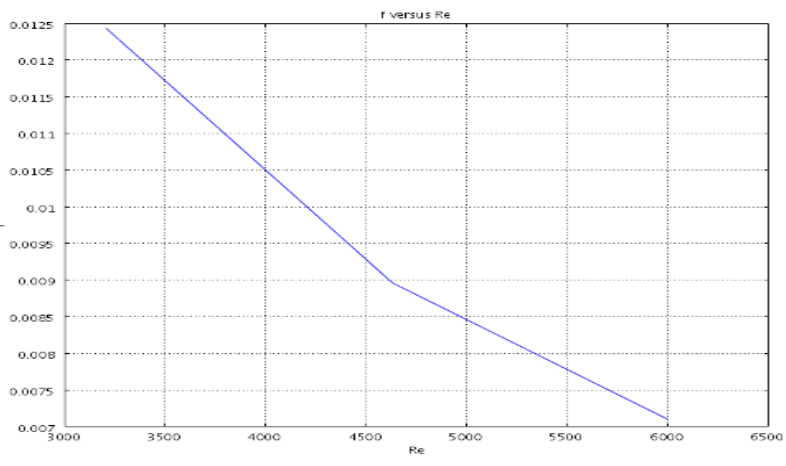

Fig 6: Computed friction factor verses Reynolds number at equal inlet and wall temperature $T_{i n}=T_{w}=60^{\circ} \mathrm{c}$.

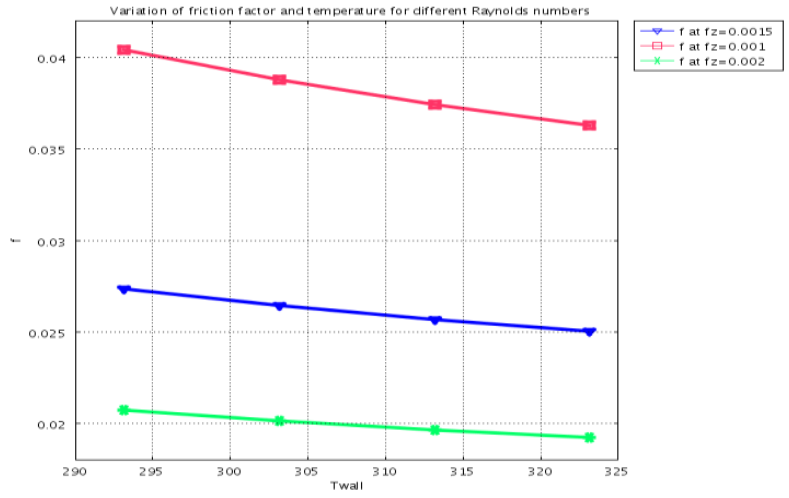

(a)

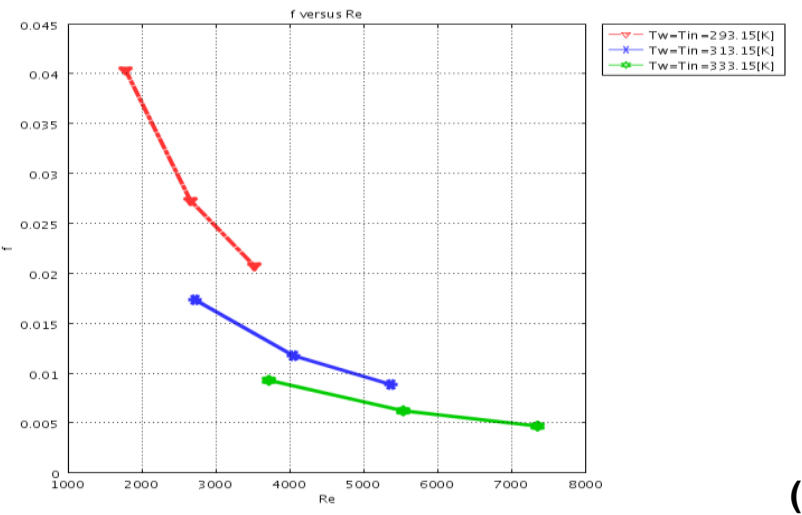

b)

Fig 7: (a) Computed Friction factors Vrs. Reynolds number, (b) Computed friction factors Vrs Reynolds number.

In Fig 7(a) showing the effect of pressure gradient on the friction factor at varying temperatures, we observe that the friction factor decreases monotonically with increasing pressure gradient. Therefore the friction factor increases with 
decreasing period length ( $L$ ).

The effect of heat load on the friction factor is shown in Fig 7(b).

It is clear that as temperature increases the transition from laminar to turbulent is lower than in conventional flows and the friction factor is reduced since the effect of heat load reduces viscous force in the flow.

There are interesting periodic pressure patterns shown in Fig 8(b). Two observations are clear: the first is the high dominating pressure zone to the left on the crest of the corrugations and the low pressure zone to the right on the trough of the corrugation. The normal to the separation point of this high and low pressure zone from the side of the crest makes an angle of about $80^{\circ}$ to the flow, this is an indication of the force exerted by the wall to slow down the flow in the axial direction.

The smaller the angle the higher the force of the wall on the flow and this force is high when the corrugation sizes increase. In the low pressure zone the momentum is lower than the free stream and the velocity near the wall reduces. This creates a flow reversal and a region of recirculation as shown in Fig $8(a)$. The second observation is that looking closely at the three periods in Fig 8 (b) we see clear instabilities in the solution on the bottom left and upper left corners of the first and third periods respectively, whiles the solution in the second period is smooth. For this reason we introduced this model to take care of the instabilities in the pressure flow. Fig 8(a) shows a typical solution of colored surfacevelocity, arrows- velocity field, yellowish linesstreamlines.

Periodic boundary condition for Temperature: We formulate periodic boundary condition for the temperature as we did for the pressure and velocity to find its effect on the flow. Let the temperature be defined as:

$T=T(r, z)+\tau z$

and

$\Delta T=T_{2}-T_{1}$

For periodicity,

$T\left(r, z_{1}\right)=T\left(r, z_{2}\right)$

Then
$\Delta T=\tau\left(z_{2}-z_{1}\right)$

Therefore the periodic boundary condition is given by:

$T\left(r, z_{\text {in }}\right)=T\left(r, z_{\text {out }}\right)+\tau L$

The numerical results for prescribing periodic boundary conditions for the velocity, pressure and temperature are shown in Fig 9. In Fig 9 and Fig 8(b) we notice that if the flow is fully developed, then the temperature of the fluid is equal everywhere. Thus it does not depend on whether the temperature is periodic or not.

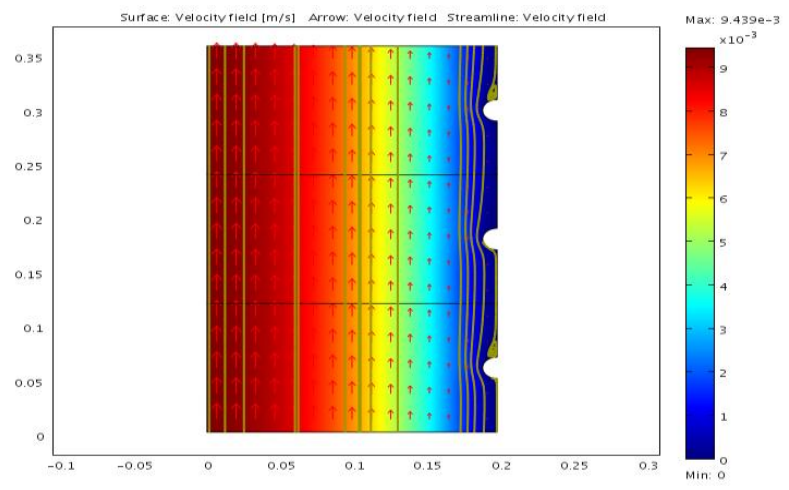

(a)

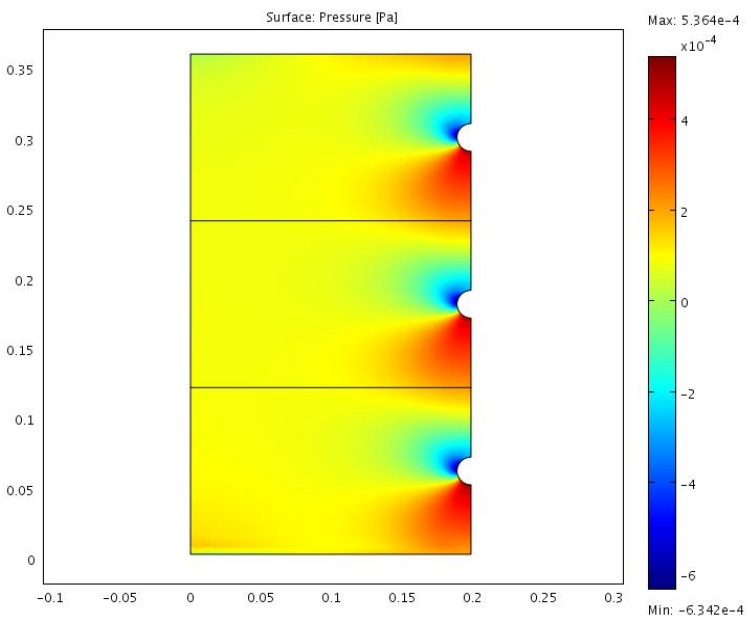

(b)

Fig 8: (a) Colored surface-velocity, arrowsvelocity field, yellowish lines-streamlines, (b) The periodic pressure. 


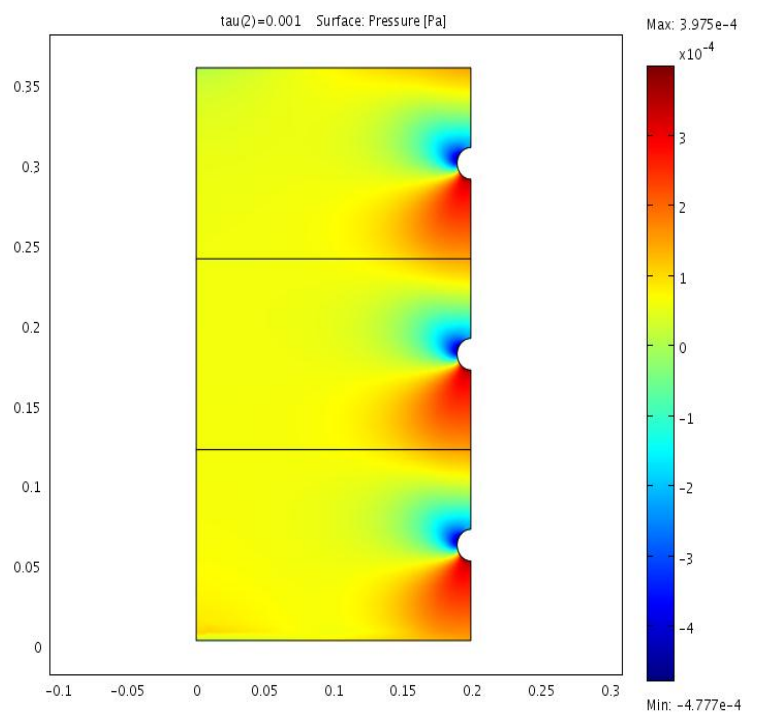

Fig 9: Numerical solution periodic pressure at $T_{\text {in }}=60 \mathrm{~K} ; T_{w}=200 \mathrm{~K}$.

Fig 10 shows the effect of heat load on the friction factor at equal and unequal inlet and wall temperatures. It can be noticed that a large difference between the inlet and wall temperatures give a significant increase of the friction factor likewise when the temperature is low.

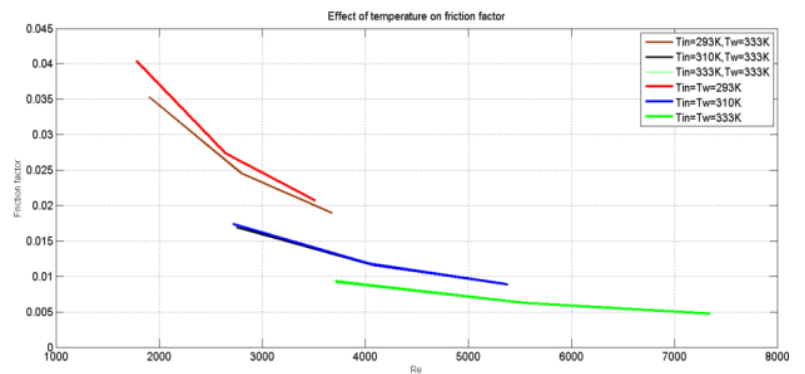

Fig 10: Effect of temperature on friction factor.

Computations from the model can be used to calculate the input power of a pump required to push a fluid through one period of the pipe as follows:

$$
\begin{aligned}
\text { Input power } & =\frac{\rho Q H}{\eta}, \text { where } \\
\mathrm{H} & =\Delta \mathrm{p}+\mathrm{h}_{f} \\
h_{f} & =\frac{f L \overline{v^{2}}}{2 g D}
\end{aligned}
$$

where $\eta$ is the efficiency of the pump, $H$ is the total head lose and $h_{f}$ is the frictional head lose, $Q$ is the flow rate. Then the input power can be written as:

Input power $=\frac{\pi \Delta p D^{4}}{128 \eta}\left[\frac{\rho g \Delta p}{\mu L}+\frac{\mu f \mathrm{Re}^{2}}{2 \rho D^{3}}\right]$

In equation (0.19), the product of the friction factor and the square of the Reynolds number $\left(f \mathrm{Re}^{2}\right)$, shows that heat load has a significant effect on the input power requirement of a pump.

CONCLUSION: The effect of heat load on the friction factor for fluid flow in a corrugated pipe has been effectively and efficiently computed from the coupled momentum and energy equations. We have used the idea of quasi-periodicity and periodic boundary conditions on a fully developed flow to simulate a single period of the corrugations as a true representative of an infinitely long pipe. Meanwhile we have shown in the analysis that when the flow is fully developed, it is independent of periodic boundary conditions for the temperature. Pressure flows are usually characterized by adverse pressure gradients and instabilities caused by shear stresses but with the introduction of the new geometry for such flows we obtained smooth solutions in the second domain or period. The curves in Figures $(6,7$, and 10) give a good fit for the effect of heat load on the friction factor which cannot be found on the Moody Diagram. Engineers working in areas of high temperatures can use these plots and the Darcy Weisbach equation to calculate frictional head loss for a given flow rate of a special fluid through a pipe with known diameter, length and roughness. Finally the increase in heat load on the pipe wall or the fluid changes the transition from laminar to turbulent flow drastically and the friction factor has been shown to decrease monotonically with increasing heat load.

\section{REFERENCES}

Balasubramanian R., Rame E., Kizito J., and Kassemi M. (2006). Two Phase flow modeling: Summary of flow regimes and pressure drop correlations in reduced and partial gravity, Report, National center for space exploration research, Cleveland, Ohio.

Beer H., Russ G. (1997). International Journal of Heat and Mass Transfer, Elsevier Volume 40, Issue 5, pp. 10611070.

Comsol multiphysics. http://www.comsol.com/.

Graetz L. (1885). Ann.Physik, 25, 337

Hanke M.,( 2006). Short introduction to comsol multiphysics.

Latif M. J.,( 2006). Heat Convection. Springer-Verlarg Berlin, Heidelberg. 
Mahmud S., Sadrul A. K. M. I., Foroz C. M. (2003) Flow and heat transfer characteristics inside a wavy tube.

Moody L. F. (1944). Friction factors for pipe flow, Princeton N.J.

Nyarko P. R. (ir) (2011). Modeling The Effects of Heat Load on The Friction Factor in Corrugated Hoses, Lambert Academic Publishing,

Patankar S. V., Liu C., and Sparrow E. M. (1977). Fully developed flow and heat transfer in ducts having streamwise-periodic variations of cross-sectional area. J. Heat Transfer

Pisarenco M. (2007). Friction factor estimation for tubulent flows in corrugated pipes with rough walls, Master Thesis TU/e Eindhoven.

Pitts D. R., Leighton E. S., (1997). Heat Transfer, second edditon. P.E. New York, Mc-Graw Hill.

Presziosi L., Chen K., and Joseph D.D., (1989). Lubricated pipelining: stability of core- annular flow. J. Fluid Mec., 201:323-356.

Schenk O. and Gartner K. (2004). Solving unsymmetric sparse systems of linear equations with PARDISO. Journal of Future Generation Computers Systems. 20(3): 475-487.
Schenk O. and Gartner K. (2006). On fast factorization pivoting methods for symmetric indefinite systems, 23:158-179.

Schlicting H., Gersten K. (2000). Boundary Layer Theory, 8th Revised and Enlarged Edition (English). SpringerVerlag, New York.

Subbarao P. M.V. (2010) Analysis of convection in internal flows.

Van der Linden B. J., Ory E., Dam J. A. M., Tijsseling A. S., Pisarenco M. (2009) Efficient computation of threedimensional flow in helically corrugated hoses including swirl,

Wang G., Vanka S. P. (1995). Convective heat transfer in periodic wavy passages, Elsevier Volume 38, Issue 17, pp. 3219-3230.

White F.M. (2003). Fluid Mechanics fifth edition, Boston Mass, WCB McGrew-Hill, pp 238.

White R., (2008). Comsol acoustics introduction, Tufts University, School of Engineering 